\title{
The Significance of the Expression of FBXO31 in Gastric Cancer"
}

\author{
Tomoya Sudo $^{1,2}$, Ryunosuke Kogo ${ }^{1}$, Naohiro Nishida ${ }^{1}$, Keisuke Takahahi ${ }^{1}$, Genta Sawada ${ }^{1}$, \\ Masahisa Ishibashi ${ }^{1}$, Junji Kurashige ${ }^{1}$, Ryutaro Uchi ${ }^{1}$, Tae Matsumura ${ }^{1}$, Hiroki Ueo ${ }^{1}$, \\ Kousuke Mima ${ }^{1}$, Sayuri Akiyoshi' ${ }^{1}$, Keishi Sugimachi' ${ }^{1}$, Kouhei Shibata ${ }^{1}$, Hiromasa Fujita ${ }^{2}$, \\ Kazuo Shirouzu', Masaki Mori ${ }^{3}$, Koshi Mimori ${ }^{1 \#}$
}

${ }^{1}$ Department of Surgery, Medical Institute of Bioregulation, Kyushu University, Beppu, Japan; ${ }^{2}$ Department of Surgery, Kurume University School of Medicine, Kurume, Japan; ${ }^{3}$ Department of Surgical Oncology, Graduate School of Medicine, Osaka University, Osaka, Japan.

Email: "kmimori@beppu.kyushu-u.ac.jp

Received November $14^{\text {th }}, 2012$; revised December $15^{\text {th }}, 2012$; accepted December $26^{\text {th }}, 2012$

\begin{abstract}
Loss of Heterozygosity (LOH) is commonly considered to be one of a reason when some genes lose their function. Numbers of tumor suppressor genes are existing on the LOH lesion and chromosome 16q24 had been reported as a LOH region in gastric cancer. Little is known about what kind of tumor suppressor genes locates around the position. F-box protein, (FBXO31) is a candidate tumor suppressor gene encoded in chromosome 16q24.3 and LOH of the gene was reported in breast cancer, hepatocellular carcinoma and ovarian cancer but the status of FBXO31 was not analyzed in gastric cancer so far. One hundred twenty-seven pairs of tumor and corresponding normal tissue specimens collected from gastric cancer patients who underwent gastrectomy. Total RNAs were extracted from those samples and the expression of FBXO31 was investigated using real time quantitative RT-PCR analysis. Patients were classified into FBXO31 high expression group and low expression group. Clinicopahological factors were compared between the two groups and importance of FBXO31 was investigated. The standardized expression of FBXO31 was not significantly different between tumor $(0.43 \pm 0.46)$ and the corresponding $0.49 \pm 0.55$ in normal tissue $(p=0.39)$. Two years survival rate was $77 \%$ in FBXO31 high expression group and 54\% in low expression group however the chance of survival rate of high expression group was dropped in 5 years (Wilcoxon $p=0.01$ ). Clinicopathological factors were compared between the two groups and peritoneal dissemination was observed significantly higher in FBXO31 low expression group than did in high expression group $(p=0.0398)$. In order to predict existence of peritoneal dissemination of gastric cancer before surgery, FBXO31 may become a favorite marker for the low risk of peritoneal dissemination.
\end{abstract}

Keywords: FBXO31; LOH; Gastric Cancer

\section{Introduction}

Gastric cancer is the second leading cause of cancer death in world wide and the ratio was particularly high in eastern Asia [1]. In Japan, modification of diagnostic procedure and upgrading of therapeutic techniques made it possible to remarkably improve the prognosis of the disease and the recent overall five years survival and recurrence free survival rate were accounted for $70 \%$ and $63 \%$ respectively. However, gastric cancer could not be completely cured yet and thousands of newly diagnosed patients lose their lives due to the disease.

\footnotetext{
${ }^{*}$ This work was supported in part by the following grants and foundations: Grant-in-Aid for Scientific Research: 21679006, 20390360, 20590313, 20591547, 21591644, 21592014, 20790960, 21791297, 21229015,20659209 , and 20012039.

\#Corresponding author.
}

The surgical strategy is aggressively modified and the extensive surgery such as D2 lymphnode dissection plus para-aortic nodal dissection was tried out however statistical significance could not be achieved comparing with D2 lymphadencetomy alone [2]. Therefore, it will be up to the development of non-surgical treatment if there is a further room for the more modification of gastric cancer therapy. S-1 based chemotherapy was demonstrated to be efficient as an adjuvant chemotherapy for stage II or III gastric cancer patients [3] although the effect was not sufficient against far advanced gastric cancer thereby novel therapeutic arms such as molecular targeting drugs should be developed.

Cyclin D1 is one of a key driving transcription factors associated with oncogenesis in gastric cancer [4] and Hirata et al. identified that Helicobactor pylori activates 
Cyclin D1 promoter and induces cellular proliferation [5]. On the other hand, F-box protein, FBXO31 is a candidate tumor suppressor gene that controls cell cycle through cyclin D1 regulation. Santra et al. demonstrated that FBXO31 comprises SCF complex with SKP1 and CUL1 and the SCF complex binds to cyclin D1 then induces cyclin D1 degradation through ubiqutination and that lead to G1 arrest [6]. Loss of heterozygosity (LOH) is considered to be primary reason for functional loss of FBXO31. LOH in chromosome 16q24.3 was reported in breast cancer [7], ovarian cancer [8], liver cancer [9] and prostate cancer [10]. Mori et al. identified that chromosome $16 \mathrm{q} 24$ is frequently deleted in human gastric cancer using $\mathrm{LOH}$ analysis and they concluded that some tumor suppressor genes in 16q24.1-q24.3 [11] but actual expression status of FBXO31 is not fully investigated in gastric cancer. Purpose of this study is to examine the expression status of cyclin D1 and FBXO31 and investigate significance the expression of $\mathrm{FBXO} 31$ in gastric cancer.

\section{Materials and Methods}

\subsection{Clinical Tissue Samples}

One hundred twenty-seven patients with gastric cancer who underwent surgery at our institutes were entered in this study. The resected tumor and paired non-tumor tissue specimens were immediately frozen in liquid nitrogen and kept at minus $80^{\circ} \mathrm{C}$ until analysis. Frozen tissue specimens were homogenized in guanidinium thiocyanate, and total RNAs were obtained by ultracentrifugation through a cesium chloride cushion as described previously $[12,13]$. Written informed consent was obtained from all patients. All patients were closely followed after surgery at regular one-month intervals.

\subsection{Real-Time Quantitative RT-PCR}

Sense and anti-sense primers encoding the middle portion of the FBXO31 gene located on chromosome, including intron and exon sequences, were designed and constructed. The sequences of the FBXO31 primers were as follows: sense primer 5'-AATCCGGCCTTTTGACCAGA-3'; antisense primer 5'-TCCGCTCACAGGAAGAGCAC-3' as described previously [6]. Glyceraldehyde-3-phosphate dehydrogenase (GAPDH) was used as an internal control. The sequences of the GAPDH primers were as follows: sense 5'-TGAACGGGAAGCTCACTGG-3'; antisense 5'-TCCACCACCCTGTTGCTGTA-3'. Real-time monitoring of the PCR reactions was performed using the LightCyclerTM system (Roche Applied Science, Indianapolis, IN, USA) and SYBR green I dye (Roche Diagnostics). Monitoring was performed according to the manufacturer's instructions, as described previously [14]. In brief, a master mixture was prepared on ice containing $1 \mu \mathrm{l}$ of cDNA of each gene, $2 \mu \mathrm{l}$ of LC DNA Master SYBR Green I mix, 50 ng of primers, and $2.4 \mu \mathrm{l}$ of $25 \mathrm{mM} \mathrm{MgCl}$. The final volume was then adjusted to $20 \mu \mathrm{l}$ with water. After the reaction mixture was loaded into the glass capillary tube, PCR was carried out under the following cycling conditions: initial denaturation at $95^{\circ} \mathrm{C}$ for $10 \mathrm{~min}$, followed by 35 cycles of denaturation at $95^{\circ} \mathrm{C}$ for $1 \mathrm{~min}$, annealing at $56^{\circ} \mathrm{C}$ for $1 \mathrm{~min}$ and extension at $72^{\circ} \mathrm{C}$ for $1 \mathrm{~min}$. After amplification, the products were subjected to a temperature gradient from $68^{\circ} \mathrm{C}$ to $95^{\circ} \mathrm{C}$ at $0.2^{\circ} \mathrm{C} / \mathrm{s}$ under continuous fluorescence monitoring to produce a melting curve of the products. We determined the expression levels of FBXO31 and GAPDH by comparison with control human cDNA. The fit point method was employed to determine the cycle in which the log-linear signal was first distinguishable from the baseline, and that cycle number was then used as a crossing-point value. The standard curve was produced by measuring the crossing point of each standard value and plotting them against the logarithmic value of concentration. The FBXO31 concentration of each sample was calculated by plotting their crossing points against the standard curve. FBXO31 concentration was then divided by the concentration of endogenous reference (GAPDH) to obtain normalized FBXO31 expression [15]. Each assay was performed three times to verify the results, and the mean mRNA expression was used for the statistical analysis.

\subsection{Classification Criteria}

The expression level of FBXO31 was calculated as follows. The standardized expression of FBXO31 in tumor tissue was divided by that of FBXO31 in corresponding normal tissue and if the calculated number was equal to or more than the mean value of 0.97 was classified as high group and less than the number was classified as low group.

\subsection{Immunohistochemical Analysis}

Several formalin-fixed and paraffin-embedded tissue sections corresponding to the samples used for mRNA expression analysis were analyzed. Tissue sections were de-paraffinized, soaked in $0.01 \mathrm{M}$ sodium cytorate buffer, and boiled in an electronic oven for 15 minutes at 500 watts to retrieve cell antigens. The tissue sections were immunohistochemically stained using the streptavidinbiotin peroxydase method (Universal Dako Cytomation LSAB $^{\circledR}$ kit; Dako, Kyoto, Japan) with a primary antibody against FBXO31 (rabbit polyclonal antibody; ABGENT, San Diego, USA). In brief, the sections were blocked by $3 \% \mathrm{H}_{2} \mathrm{O}_{2}$ for 5 minutes and incubated overnight with primary antibody at $4^{\circ} \mathrm{C}$. The samples were 
then washed with TBS buffer and subsequently incubated with secondary antibody for 30 minutes.

\subsection{Statistical Analysis}

The data obtained from real-time RT-PCR and the patients' clinicopathological variables were processed by the statistical software JMP (SAS Institute, Inc., Cary, NC, USA). The Student's t-test and the Chi-squared test with Yates' correction factor were used. The postoperative disease-free survival rate was calculated by the Kaplan-Meier method, and the differences in survival between the groups were compared using the log-rank test. The findings were considered to be significant when their p-value was less than 0.05 .

\section{Results}

\subsection{Short-Term Survival Was Better in FBXO31 High Expression Group}

The expression of FBXO31 was investigated using cDNA samples collected from gastric cancer tissues and corresponding normal tissues. The standardized median expression of FBXO31 was $0.40 \pm 0.04$ in tumor and $0.43 \pm 0.04$ in normal tissue respectively. There was no significant difference of FBXO31 expression between tumor and normal tissue. The gastric cancer patients were divided into high expression group and low expression group. The classification was performed following the procedure being mentioned in the materials and methods. As shown in Figure 1, two year survival was $77 \%$ in FBXO31 high expression group on the other hand 54\% in FBXO31 low expression group. The short term survival was significantly better in FBXO31 high expression group than did in low expression group (Wilcoxon $\mathrm{p}=$ 0.01). However the advantage was narrowed when it was compared in five year survival (Logrank $p=0.08$ ). To investigate the importance of FBXO31 in gastric cancer, the expression of FBXO31 and clinicopathological factors were compared. As indicated in Table 1, the expression of FBXO31 was statistically correlated with peritoneal dissemination and the percentage was significantly lower in FBXO31 high expression group than did in low expression group. There was no correlation between the expression of FBXO31 and the other clinicopathological factors.

\subsection{Correlation between the Expression of FBXO31 and the Expression of Cyclin D}

To investigate association between FBXO31 and cyclin $\mathrm{D}$ in gastric cancer, immunohistochemical staining was performed. The expression of FBXO31 was expressed in the nucleus of normal epithelial cell whereas cyclin D was not stained in the normal epithelial cells (Figure 2(a)). In contrast, the expression of FBXO31 was decreased and the expression of cyclin D was significantly gained in the nucleus of cancer cells (Figure 2(b)). The correlation between the expression of FBXO31 and cyclin D were investigated using qRT-PCR, however significant correlation could not be detected in RNA expression level (data not shown).

\section{Discussion}

Cyclin D1 is one of an important oncoprotein which is being frequently amplified in variety of cancers [16]. Furthermore Hirata et al. indicated that Helicobacter pyloli infection induces cyclin D1 gene activation through its' promoter activation [5]. These papers are implying that cyclin D1 plays an important role in emergence of gastric cancer. FBXO31 is one of a key regulator of cyclin D1 in response to DNA damage [6] so that the down-regulation of FBXO31 should be quite important step while occurrence of gastric cancer.

As shown in immunohistochemical staining, the pro-

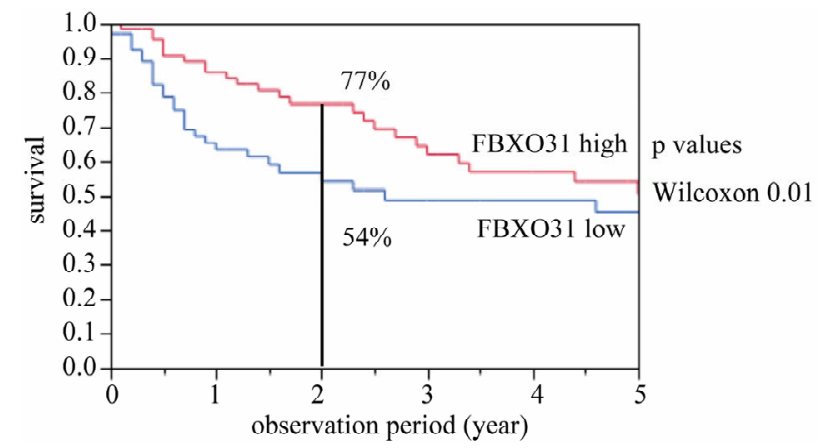

Figure 1. Kaplan-Meier curves for FBXO31 high expression group $(n=63)$ and low expression group $(n=64)$; p-value based on the Wilcoxon signed-rank test. p-value less than 0.05 was defiend statistical significance.

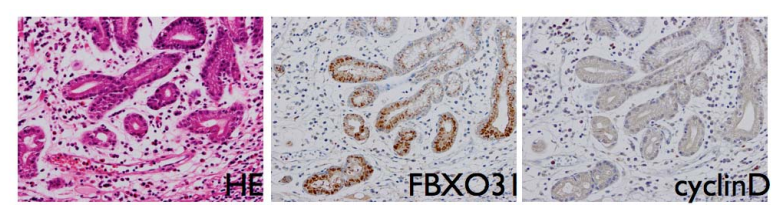

(a)

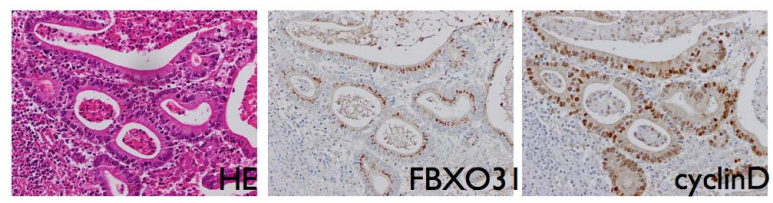

(b)

Figure 2. Represntative examples of Hematoxylin-Eosin staining and IHC analysis of FBXO31 and cyclinD of cancer sections and normal tissue sections. Upper and lower figures' magnifications are $\times 40$ respectively. (a) Normal tissue; (b) Cancerous tissue. 
Table 1. Comparison of clinicopathological factors between FBXO31 high expression group and low expression group.

\begin{tabular}{|c|c|c|c|c|}
\hline \multicolumn{2}{|c|}{ Clinicopathological factors } & \multirow{2}{*}{$\begin{array}{c}\text { High group }(\mathrm{n}=63) \\
\text { tumor factors }\end{array}$} & \multirow[t]{2}{*}{ Low group $(n=64)$} & \multirow[t]{2}{*}{ p-values } \\
\hline & & & & \\
\hline \multirow{2}{*}{ depth of tumor } & $\mathrm{T} 1-\mathrm{T} 2$ & $38(60.32 \%)$ & $35(54.69 \%)$ & \multirow{2}{*}{0.5209} \\
\hline & T3-T4 & $25(39.68 \%)$ & $29(45.31 \%)$ & \\
\hline \multirow{2}{*}{ tumor type } & types $0-2$ & 30 & 34 & \multirow{2}{*}{0.5348} \\
\hline & types 3-5 & 33 & 30 & \\
\hline \multirow{2}{*}{ lymphatic invasion } & ly0-1 & $25(39.68 \%)$ & $18(28.13 \%)$ & \multirow{2}{*}{0.1688} \\
\hline & ly2-3 & $38(60.32 \%)$ & $46(71.88 \%)$ & \\
\hline \multirow{2}{*}{ vascular invasion } & v0-1 & $49(77.78 \%)$ & $45(70.31 \%)$ & \multirow{2}{*}{0.3367} \\
\hline & v2-3 & $14(22.22 \%)$ & $19(29.69 \%)$ & \\
\hline & & metastasis & & \\
\hline \multirow{2}{*}{ lymph node metastasis } & N0-1 & $42(66.67 \%)$ & $36(56.25 \%)$ & \multirow{2}{*}{0.2279} \\
\hline & $\mathrm{N} 2-4$ & $21(33.33 \%)$ & $28(43.75 \%)$ & \\
\hline \multirow{2}{*}{ liver metastasis } & $\mathrm{HO}$ & $56(88.89 \%)$ & $59(92.19 \%)$ & \multirow{2}{*}{0.9369} \\
\hline & H1 & $7(11.11 \%)$ & $5(7.81 \%)$ & \\
\hline \multirow{2}{*}{ peritoneal dissemination } & P0 & $56(88.89 \%)$ & $48(75 \%)$ & \multirow{2}{*}{$0.0398^{*}$} \\
\hline & $\mathrm{P} 1$ & $7(11.11 \%)$ & $16(25 \%)$ & \\
\hline \multirow{3}{*}{ distant metastasis } & M0 & $61(96.83 \%)$ & $62(96.89 \%)$ & \multirow{2}{*}{0.6729} \\
\hline & M1 & $2(3.17 \%)$ & $2(3.11 \%)$ & \\
\hline & & clinical stage & & \\
\hline \multirow{2}{*}{ stage } & stages I-II & $37(58.73 \%)$ & $30(46.88 \%)$ & \multirow{2}{*}{0.2081} \\
\hline & stages III-IV & $26(41.27 \%)$ & $34(53.12 \%)$ & \\
\hline
\end{tabular}

tein level expression of FBXO31 is well observed whereas the expression of cyclin D1 was not identified in normal gastric mucosa cells (Figure 2(a)). This expression difference of cyclin D1 between cancer cells and normal mucosal cells were identical with previous papers $[4,17]$. Moreover, the expression of FBXO31 was apparently weak in the cells that expressed strong cyclin D1 (Figure 2(b)). The inverse correlation between cyclin D1 and FBXO31 was demonstrated by Santra et al. and they probed that cyclin D1 degradation results from a direct interaction with FBXO31 and is dependent on the F-box motif of FBXO31 and phosphorylation of cyclin D1 in breast cancer [6]. In this study we for the first time identified that the similar relationship was also observed in gastric cancer and FBXO31 might play a crucial role in regulation of cyclin D1 not only in breast cancer but also in variety of cancers including gastric cancer.

In the level of mRNA expression, we could not confirm that significant down regulation of FBXO31 in cancerous tissue comparing with normal tissue. The cDNA library we used in this study was bulky samples excised from gastric cancer patients so that some amount of normal tissues were possibly contaminated. The actual down regulation of FBXO31 might be complemented by the expression of normal tissue. Laser micro dissection should be used to analyze the exact mRNA expression difference between normal and cancer tissue.

The overall survival of FBXO31 high expression group was significantly better than that of low expression group in short term period but the advantage was discarded later (Figure 1). This result may show that the regulation of cyclin D1 by FBXO31 is effective in early event of the tumor progression however, the tumor progression gradually being supported by other factors. Wang et al. revealed the existence of numbers of somatic mutations in gastric cancer using next-generation sequencing analysis and they predicted the driver genes including TP53, PTEN etc. [18]. Disruption of those genes takes place for cyclinD1 so that the preventive effect of FBXO31 may be perished. By analyzing the expression of FBXO31, we possibly detect the patient who has high risk of the existence of peritoneal dissemination. As a result of that, the expression FBXO31 would be beneficial biological marker to determine a treatment strategy before surgery.

\section{Acknowledgements}

We thank Mr. M. Kasagi and Ms. T. Shimooka for extraordinary assistance with RT-PCR and immunohistochmical staining. 


\section{REFERENCES}

[1] P. Pisani, D. M. Parkin, F. Bray and J. Ferlay, "Estimates of the Worldwide Mortality from 25 Cancers in 1990," International Journal of Cancer, Vol. 83, No. 1, 1999, pp. 18-29.

[2] M. Sasako, T. Sano, S. Yamamoto, Y. Kurokawa, A. Nashimoto, A. Kurita, et al., "D2 Lymphadenectomy Alone or with Para-Aortic Nodal Dissection for Gastric Cancer," The New England Journal of Medicine, Vol. 359, No. 5, 2008, pp. 453-462. doi:10.1056/NEJMoa0707035

[3] S. Sakuramoto, M. Sasako, T. Yamaguchi, T. Kinoshita, M. Fujii, A. Nashimoto, et al., "Adjuvant Chemotherapy for Gastric Cancer with S-1, an Oral Fluoropyrimidine," The New England Journal of Medicine, Vol. 357, No. 18, 2007, pp. 1810-1820. doi:10.1056/NEJMoa072252

[4] D. S. Arici, E. Tuncer, H. Ozer, G. Simek and A. Koyuncu, "Expression of Retinoblastoma and Cyclin D1 in Gastric Carcinoma," Neoplasma, Vol. 56, No. 1, 2009, pp. 63-67. doi:10.4149/neo 20090163

[5] Y. Hirata, S. Maeda, Y. Mitsuno, M. Akanuma, Y. Yamaji, K. Ogura, et al., "Helicobacter Pylori Activates the Cyclin D1 Gene through Mitogen-Activated Protein Kinase Pathway in Gastric Cancer Cells," Infection and Immunity, Vol. 69, No. 6, 2001, pp. 3965-3971. doi:10.1128/IAI.69.6.3965-3971.2001

[6] M. K. Santra, N. Wajapeyee and M. R. Green, "F-Box Protein FBXO31 Mediates Cyclin D1 Degradation to Induce G1 Arrest after DNA Damage," Nature, Vol. 459, No. 7247, 2009, pp. 722-725. doi:10.1038/nature08011

[7] R. Kumar, P. M. Neilsen, J. Crawford, R. McKirdy, J. Lee, J. A. Powell, et al., "FBXO31 Is the Chromosome 16q24.3 Senescence Gene, a Candidate Breast Tumor Suppressor, and a Component of an SCF Complex," Cancer Research, Vol. 65, No. 24, 2005, pp. 11304-11313. doi:10.1158/0008-5472.CAN-05-0936

[8] V. Launonen, A. Mannermaa, F. Stenbäck, V.-M. Kosma, U. Puistola, P. Huusko, et al., "Loss of Heterozygosity at Chromosomes 3, 6, 8, 11, 16, and 17 in Ovarian Cancer: Correlation to Clinicopathological Variables," Cancer Genetics and Cytogenetics, Vol. 122, No. 1, 2000, pp. 49-54. doi:10.1016/S0165-4608(00)00279-X

[9] H. L. Huang, W. L. Zheng, R. Zhao, B. Zhang and W. L. Ma, "FBXO31 Is Down-Regulated and May Function as a Tumor Suppressor in Hepatocellular Carcinoma," Oncology Reports, Vol. 24, No. 3, 2010, pp. 715-720.

[10] P. Härkönen, A. P. Kyllönen, S. Nordling and P. Vihko,
"Loss of Heterozygosity in Chromosomal Region 16q24.3 Associated with Progression of Prostate Cancer," The Prostate, Vol. 62, No. 3, 2005, pp. 267-274. doi:10.1002/pros.20147

[11] Y. Mori, M. Matsunaga, T. Abe, S. Fukushige, K. Miura, M. Sunamura, et al., "Chromosome Band 16q24 Is Frequently Deleted in Human Gastric Cancer," British Journal of Cancer, Vol. 80, No. 9, 1999, pp. 556-562. doi:10.1038/sj.bjc. 6690391

[12] M. Mori, K. Mimori, H. Inoue, G. F. Barnard, K. Tsuji, S. Nanbara, et al., "Detection of Cancer Micrometastases in Lymph Nodes by Reverse Transcriptase-Polymerase Chain Reaction," Cancer Research, Vol. 55, No. 15, 1995, pp. 3417-3420.

[13] T. Utsunomiya, H. Inoue, K. Taguchi, M. Shimada, K. Sugimachi and M. Mori, "G Protein Gamma 7 Expression as a New Clinicopathological Marker in Patients with Intrahepatic Cholangiocarcinoma," Archives of Surgery, Vol. 137, No. 3, 2002, pp. 181-185.

[14] K. Ogawa, T. Utsunomiya, K. Mimori, Y. Tanaka, F. Tanaka, H. Inoue, et al., "Clinical Significance of Elongation Factor-1 Delta mRNA Expression in Oesophageal Carcinoma," British Journal of Cancer, Vol. 91, No. 22, 2004, pp. 282-286. doi:10.1038/sj.bjc.6601941

[15] I. Bièche, P. Onody, I. Laurendeau, M. Olivi, D. Vidaud, R. Lidereau and M. Vidaud, "Real-Time Reverse Transcription-PCR Assay for Future Management of ERBB2Based Clinical Applications," Clinical Chemistry, Vol. 45, No. 8, 1999, pp. 1148-1156.

[16] P. Schraml, J. Kononen, L. Bubendorf, H. Moch, H. Bissig, A. Nocito, et al., "Tissue Microarrays for Gene Amplification Surveys in Many Different Tumor Types," Clinical Cancer Research, Vol. 5, No. 8, 1999, pp. 19661975.

[17] G. K. Anagnostopoulos, D. Stefanou, E. Arkoumani, J. Karagiannis, K. Paraskeva, L. Chalkley, et al., "Immunohistochemical Expression of Cell-Cycle Proteins in Gastric Precancerous Lesions," Journal of Gastroenterology and Hepatology, Vol. 23, No. 4, 2008, pp. 626-631. doi:10.1111/j.1440-1746.2007.05219.x

[18] K. Wang, J. Kan, S. T. Yuen, S. T. Shi, K. M. Chu, S. Law, et al., "Exome Sequencing Identifies Frequent Mutation of ARID1A in Molecular Subtypes of Gastric Cancer," Nature Genetics, Vol. 43, No. 12, 2011, pp. 1219-1223. doi:10.1038/ng.982 